\title{
Microphase Separation: Enabling Isosorbide-Based Polycarbonates with Improved Property Profile
}

Jan Henk Kamps, ${ }^{*}, \dagger+\downarrow$ Vaidyanath Ramakrishnan, ${ }^{\dagger}$ Theo Hoeks, ${ }^{\dagger}$ Bernardus J.P. Jansen, ${ }^{\dagger}$ Rint P. Sijbesma, ${ }^{\ddagger}$ Johan P.A. Heuts

${ }^{\dagger}$ SABIC, Plasticslaan 1, 4612PX Bergen op Zoom, the Netherlands

Laboratory of Supramolecular Polymer Chemistry, Department of Chemical Engineering and Chemistry, Eindhoven University of Technology, P.O. Box 513, 5600 MB Eindhoven,

The Netherlands

*corresponding author, j.h.kamps@tue.nl, ORCID 0000-0003-0435-0764

\section{Contents}

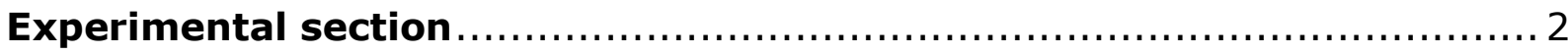

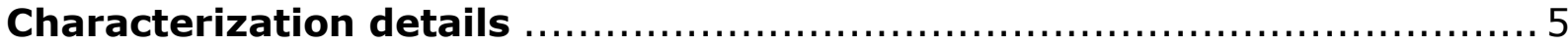

Results and discussion: Additional figures and charts ...................... 9

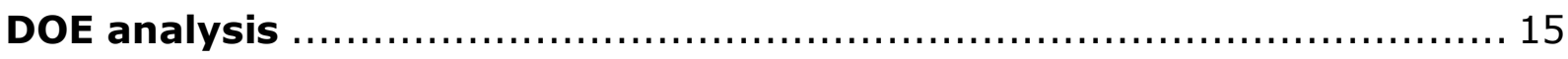

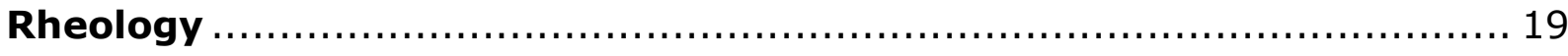




\section{Experimental section}

Example formulation for batch synthesis of isosorbide homopolycarbonate. glass reactor equipped with a mechanical stirrer and a vacuum system was charged with $8.76 \mathrm{~g}$ of isosorbide (0.0599 mole) and $20.00 \mathrm{~g}$ of BMSC (0.0605 mole), resulting in a molar ratio of reactants of 1.01 .

Catalyst solution was prepared by adding $0.120 \mathrm{~g}$ of a $\mathrm{NaOH}$ stock solution $(0.5 \mathrm{M})$ and $4.77 \mathrm{~g}$ of TBPA (40\%) to a $20 \mathrm{~mL}$ flask and fill with Milli-Q grade water to $20.00 \mathrm{~mL}$, which is added as $100 \mu \mathrm{L}$ quantity to the reaction mixture.

Example formulation for batch synthesis of isosorbide / pri1009 (20\%)

copolycarbonate. A glass reactor equipped with a mechanical stirrer and a vacuum system was charged with $8.23 \mathrm{~g}$ of isosorbide (0.0563 mole), $2.06 \mathrm{~g}$ of pri1009 (3.63 millimole, $20 \mathrm{wt} \%)$ and $20.00 \mathrm{~g}$ of BMSC (0.0605 mole), resulting in a molar ratio of reactants of 1.01 .

Catalyst solution was prepared by adding $0.120 \mathrm{~g}$ of a $\mathrm{NaOH}$ stock solution $(0.5 \mathrm{M})$ and $4.77 \mathrm{~g}$ of TBPA (40\%) to a $20 \mathrm{~mL}$ flask and fill with Milli-Q grade water to $20.00 \mathrm{~mL}$, which is added as $100 \mu \mathrm{L}$ quantity to the reaction mixture. 
The generalized structure of C36 dimer as prepared from linoleic acid (provided by Croda, Gouda, the Netherlands) is presented in Chart SI-1, structure 6, 7 or 8 as produced from dimerization of linoleic acid.

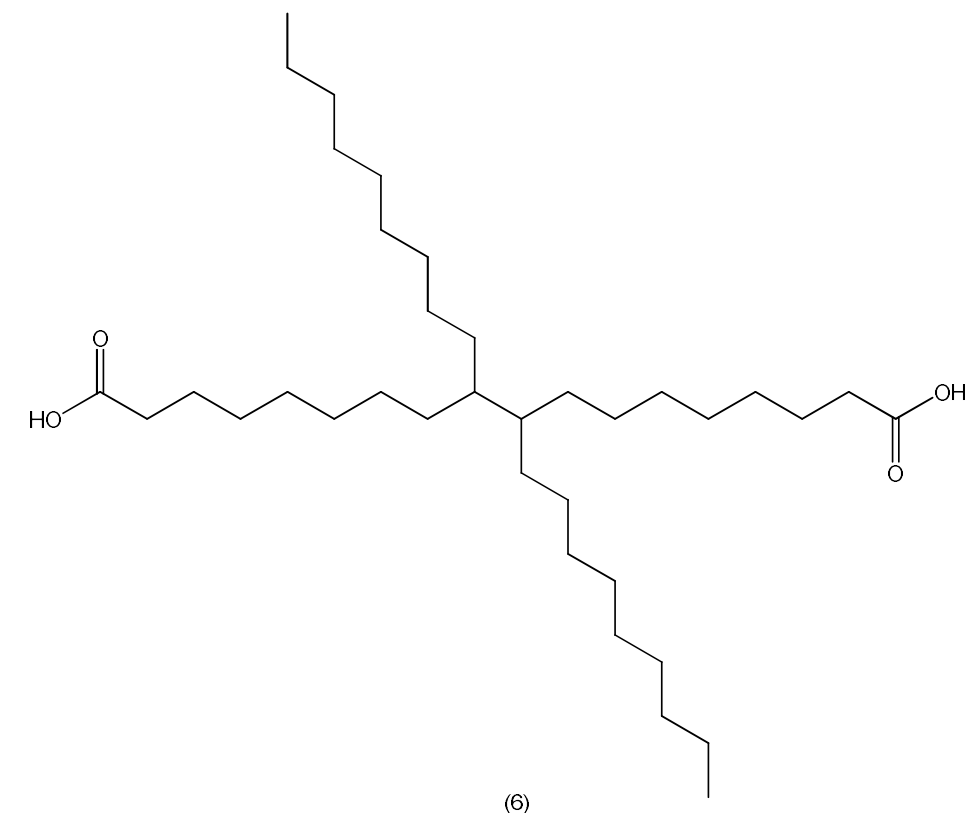<smiles>CCCCCCCCCCC1CCC(CCCCCCCC(=O)O)C(CCCCCCCC(=O)O)C1CCCCCCC</smiles>

(7)<smiles>CCCCCC1CC2CCC(CCCCCCCC(=O)O)C(CCCCCCCC(=O)O)C2CC1CCCCC</smiles>

Chart SI-1. Generalized structure of $\mathrm{C}_{36}$ dimer based on linoleic acid dimerization; linear $\mathrm{C}_{36}(6)$, cyclic $\mathrm{C}_{36}(7)$ and bicyclic $\mathrm{C}_{36}(8)$ are displayed 


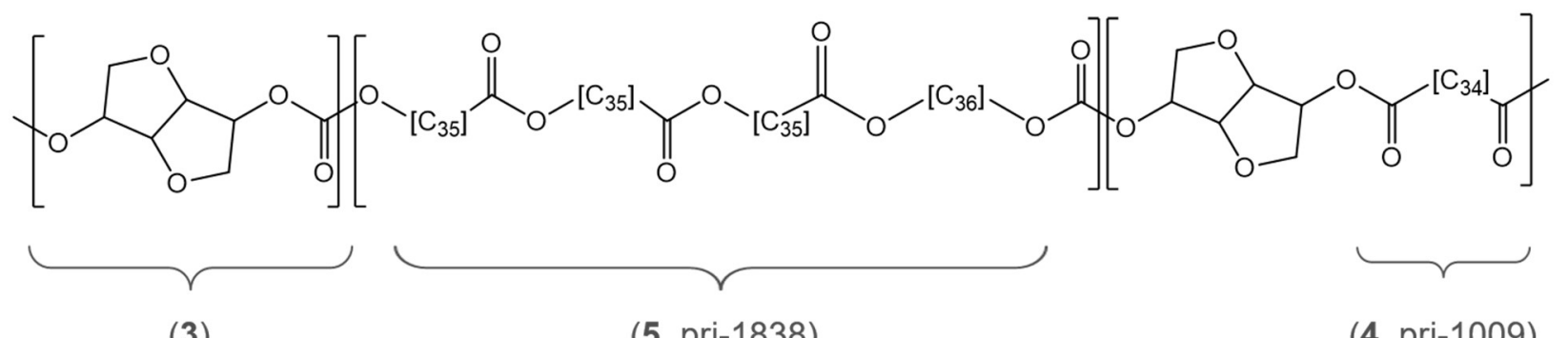

(3)

(5, pri-1838)

(4, pri-1009)

Chart SI-2. Schematic representation of isosorbide copolymer with pri-1838 (hydrogenated polyester polyol based on three $\mathrm{C}_{36}$ dimers $)$ and pri-1009 ( $\mathrm{C}_{36}$ dimer diacid) comonomers incorporated

Continuous synthesis. A 200 L stainless steel stirred tank reactor was charged with isosorbide, additional diol (or diacid) in the case of copolymer preparation, and BMSC, maintaining molar ratios of [BMSC] / [sum of diols (or diacid)] between 1 and 1.02 and charging up to $100 \mathrm{~kg}$ of monomers (Figure 1, element $\mathrm{a}_{1}$ ). A second vessel was available for alternating use, to enable continuous production of polymer (Figure 1, element $\mathrm{a}_{2}$ ). The reactor was then evacuated and purged with nitrogen three times to remove residual oxygen and then put to a constant pressure of 800 mbar. Subsequently the temperature was increased to $130{ }^{\circ} \mathrm{C}$ to melt the monomers. After a homogeneous mixture was obtained, the temperature was reduced to $100{ }^{\circ} \mathrm{C}$, and maintained for the duration of the experiment. The monomer mix was then pumped at a continuous rate $(\sim 25 \mathrm{~kg} / \mathrm{hr})$, with continuous addition of an aqueous solution of sodium hydroxide $\left(1.73 \mathrm{~g} / \mathrm{L}, 0.2 \mathrm{~mL} / \mathrm{min}, 1.5 \times 10^{-6} \mathrm{~mol} \mathrm{NaOH}\right.$ per mol diol) to the monomer mix using a HPLC pump (Figure 1, element b). The monomer mixture was then pumped through a tube fitted with static mixers. The tubular reactor was operated at $180^{\circ} \mathrm{C}-200^{\circ} \mathrm{C}$ and a pressure of $4-5$ bar (Figure 1, element c). The oligomer out of the tubular reactor was transferred to a flash devolatilization system, consisting of a pre-heater (Figure 1, element $\mathrm{d}, T=240{ }^{\circ} \mathrm{C}, p=200 \mathrm{mbar}$ ) and a flash vessel (Figure 1, element e, $T=190{ }^{\circ} \mathrm{C}, p=180$ mbar). A melt pump (Figure 1, element f) transferred the material from the flash vessel to the extruder (Figure 1, element $\mathrm{g}$ ), where the final 
polycondensation took place. The extruder, a Werner \& Pfleiderer ZSK25WLE, 25 mm 13-barrel twin-screw extruder with a $L / D=59$. The reaction mixture was extruded at a screw speed of 250 rpm. The extruder barrels were set to $270{ }^{\circ} \mathrm{C}$ and the die was set to $280{ }^{\circ} \mathrm{C}$. The extruder was equipped with five forward vacuum vents, one back-vent and was operated with one vacuum system connected to all vents ( $p \approx 1$ mbar). The methyl salicylate byproduct was removed via devolatilization through these vents. Molten strands of polymer were collected at the end of the extruder through a die and were solidified through a water bath and followed by pelletizing (Figure 1, element h).

\section{Characterization details}

Molecular weight distributions were determined by size exclusion chromatography (SEC), using an Agilent crosslinked styrene-divinylbenzene column on an Agilent 1100 chromatography set up. SEC samples were prepared in methylene chloride at a concentration of about $1 \mathrm{mg} / \mathrm{mL}$, and eluted at a flow rate of about 0.2 to $1.0 \mathrm{~mL} / \mathrm{min}$ using refractive index detection. The system was calibrated by using narrow polystyrene standards supplied by Agilent and all molecular weights reported in this article are relative to polystyrene. Nuclear magnetic resonance (NMR) spectra were measured on a Bruker Avance $400 \mathrm{MHz}$ Spectrometer at a temperature of $44{ }^{\circ} \mathrm{C}$. For each analysis, a solution of $5 \mathrm{mg} / \mathrm{mL}$ in $\mathrm{CDCl}_{3}$ with $0.03 \mathrm{wt} \% \mathrm{TMS}$ was prepared. All spectra were obtained with a spin-rate of $20 \mathrm{~Hz}$. The ${ }^{1} \mathrm{H}-\mathrm{NMR}$ measurements were performed at $400 \mathrm{MHz}$ with a $5 \mathrm{~mm}$ diameter quadruple nucleus probe, using a sweep width of $6410 \mathrm{~Hz}$ (13 to $-3 \mathrm{ppm}$ chemical shift range) with a $30^{\circ}$ flip angle, $10 \mathrm{~s}$ relaxation time, $32 \mathrm{~K}$ data points and 256 scans. Processing included apodization with $0.3 \mathrm{~Hz}$ for ${ }^{1} \mathrm{H}-\mathrm{NMR} .{ }^{13} \mathrm{C}-\mathrm{NMR}$ spectra were measured on a Bruker Avance 400 spectrometer operating at $125.75 \mathrm{MHz}$ for ${ }^{13} \mathrm{C}$. Approximately $200 \mathrm{mg}$ of the polymer sample was dissolved in $3.5 \mathrm{~mL}$ of $\mathrm{CDCl}_{3}$. The central peak of the $\mathrm{CDCl}_{3}$ resonance was used as 
an internal chemical shift standard $(=77.0 \mathrm{ppm})$. Acquisition parameters included a $30.3 \mathrm{kHz}$ spectral width and $32 \mathrm{~K}$ data points resulting in a $1.08 \mathrm{~s}$ acquisition time. A pulse delay of $1 \mathrm{~s}$ and flip angle of $45^{\circ}$ were used. Broadband proton decoupling was carried out using the Waltz-16 pulse sequence. Between 1200 and 16,000 (overnight) scans were acquired in blocks of 600 . When high resolution spectra were needed, the data was processed without any exponential apodization. A $1 \mathrm{~Hz}$ exponential apodization was applied for standard ${ }^{13} \mathrm{C}$ spectra. In addition, a polynomial baseline correction was applied. Glass transition temperatures were measured using differential scanning calorimetry (DSC) on a Perkin Elmer DSC 6, at a heating rate of $15^{\circ} \mathrm{C} / \mathrm{min}$, heat flux was recorded during the second heating scan, and the recorded heat flow curve was used to determine $T_{\mathrm{g}}$ as the midpoint of tangent lines drawn before and after the transition. The colors of molded $2.5 \mathrm{~mm}$ thick plaques were measured in the transmission mode using a Gretag-MacBeth 7000A spectrometer according to the CIE laboratory standard. The Vicat softening temperature were determined according to ISO306 using a heating rate of $120^{\circ} \mathrm{C} / \mathrm{h}$ and a force of $50 \mathrm{~N}$ on a Zwick HDT/Vicat A setup. Test specimens of $10 \mathrm{~mm} \times 10 \mathrm{~mm} \times 4 \mathrm{~mm}$ were cut from molded 80 $\mathrm{mm} \times 10 \mathrm{~mm} \times 4 \mathrm{~mm}$ ISO impact bars. Each test was repeated and the average of the two results reported. Heat deflection temperatures (HDT) were determined according to ISO75:2004 using 1.8 MPa stress on the flat surface (method A) on a Zwick HDT/Vicat A setup. Measurements were performed on molded ISO bars $(80 \times 10 \times 4 \mathrm{~mm})$ which were preconditioned at $23{ }^{\circ} \mathrm{C}$ and $50 \%$ relative humidity for 48 hours. Measurements were duplicated and the average values reported. Notched Izod impact (INI) tests were carried out according to ISO 180:2000, method A test protocol using a Zwick HIT 5.5 equipment. The tests were repeated five times on $80 \times 10 \times 3 \mathrm{~mm}$ notched molded impact bars. The test specimens were conditioned at $23{ }^{\circ} \mathrm{C}$ and $50 \%$ relative humidity for 48 hours. The impact velocity was $3.5 \mathrm{~m} / \mathrm{s}$ with a pendulum energy of $5.5 \mathrm{~J}$, the 
clamping height was $40 \mathrm{~mm}$ and the tests were conducted at $23{ }^{\circ} \mathrm{C}$. Results are reported as the average of five measurements, with standard deviation of less then $10 \%$ of the mean. Tensile modulus, stress at yield, stress at break and strain at break were determined according to ISO527 using a Zwick Z020 set up. The tensile speed was $1 \mathrm{~mm} / \mathrm{min}$ for determining the modulus and 50 $\mathrm{mm} / \mathrm{min}$ for the other properties. Samples were conditioned for 2 days at $23{ }^{\circ} \mathrm{C}$ and $50 \%$ relative humidity prior to testing. Dynamic mechanical analysis (DMA) was performed on a Rheometrics RSA2 in the bending mode (span $48 \mathrm{~mm}$ ) using an ISO impact bar. The heating rate and frequency used were $4{ }^{\circ} \mathrm{C} / \mathrm{min}$ and $1 \mathrm{~Hz}$ respectively. Transmission electron microscopy (TEM) was carried out on Tennai 12 equipment, samples cut to size using cryo-toming $\left(-110^{\circ} \mathrm{C}\right)$ followed by $\mathrm{OsO}_{4}$ (390 sec exposure) and $\mathrm{RuO}_{4}$ (390 sec exposure) staining. Experimental conditions: $100 \mathrm{kV}$, spot 2, magnification 9700x. The image analysis for particle detection and size measurement were performed using Matlab 2017b. Since contrast enhancement in the TEM images is essential for a correct particle detection and the subsequent measurement, the images were first linearly normalized within $[0,1]$ to stretch the histograms and to unify the gray values for further processing. Afterwards, a 2D median filter was applied onto each of the images for noise reduction and the particles were detected in an adaptive thresholding manner. The particle size was then characterized with the equivalent diameter, which is defined as the diameter of a circular shape giving the same area as the detected particle. Finally, all the samples were rheologically characterized using small amplitude oscillatory shear experiments with parallel plate geometries of 8.0 and $25.0 \mathrm{~mm}$ at a gap of $1 \mathrm{~mm}$, in an ARES-G2 rheometer from TA Instruments. Strain sweeps were run to determine the linear viscoelastic region and a strain of $10 \%$ was used. Simultaneous temperature-frequency sweeps were performed from $160{ }^{\circ} \mathrm{C}$ to $250{ }^{\circ} \mathrm{C}$ in a frequency range of 0.1 to $100 \mathrm{rad} / \mathrm{s}$. A correction of $2 \mu \mathrm{m} / \mathrm{K}$ was used to compensate for the thermal 
expansion of the plates. The last measurement was performed identically to the first one to check for degradation effects in the sample, as the same sample was used for different temperatures. Master curves were constructed at a reference temperature of $250{ }^{\circ} \mathrm{C}$ using the time-temperature superposition (TTS) principle. The horizontal shift factor $\left(a_{\mathrm{T}}\right)$ is given by the WLF relationship as:

$$
\log a_{T}=\frac{-C_{1}\left(T-T_{0}\right)}{C_{2}+\left(T-T_{0}\right)}
$$

where, $C_{1}$ and $C_{2}$ are empirical constants and $T_{0}$ is the reference temperature. Sometimes a vertical shift factor $\left(b_{\mathrm{T}}\right)$ is used to correct for density fluctuations. We would like to note that the copolymers containing up to $20 \%$ of soft block did not require any density corrections when shifted indicating that all samples behave as thermorheologically simple materials.

Furthermore the TTS curves are used to calculate the continuous relaxation spectrum given by the following formulas, where $H(\ln \tau)$ is the memory function and $\tau$ is the relaxation time.

$$
\begin{aligned}
& G^{\prime}(\omega)=\int_{-\infty}^{\infty} H(\ln \tau) \frac{\omega^{2} \tau^{2}}{1+\omega^{2} \tau^{2}} d \ln \tau \\
& G^{\prime \prime}(\omega)=\int_{-\infty}^{\infty} H(\ln \tau) \frac{\omega \tau}{1+\omega^{2} \tau^{2}} d \ln \tau
\end{aligned}
$$




\section{Results and discussion: Additional figures and charts}

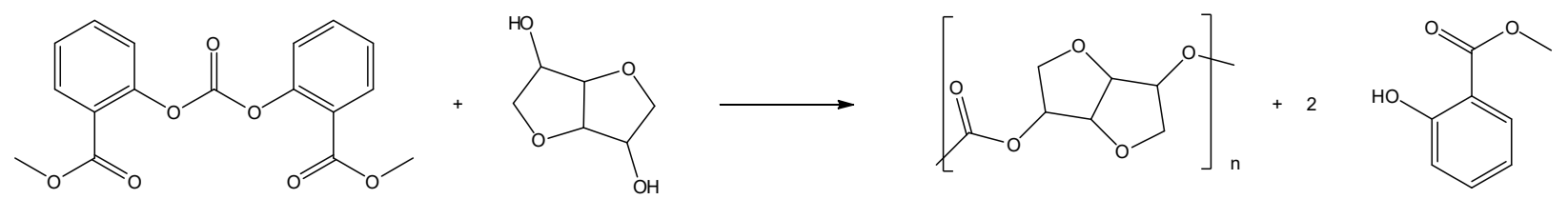

Scheme SI-1. Transcarbonation reaction between bismethylsalicyl carbonate (BMSC) and isosorbide, forming isosorbide polycarbonate and condensing off methyl salicylate

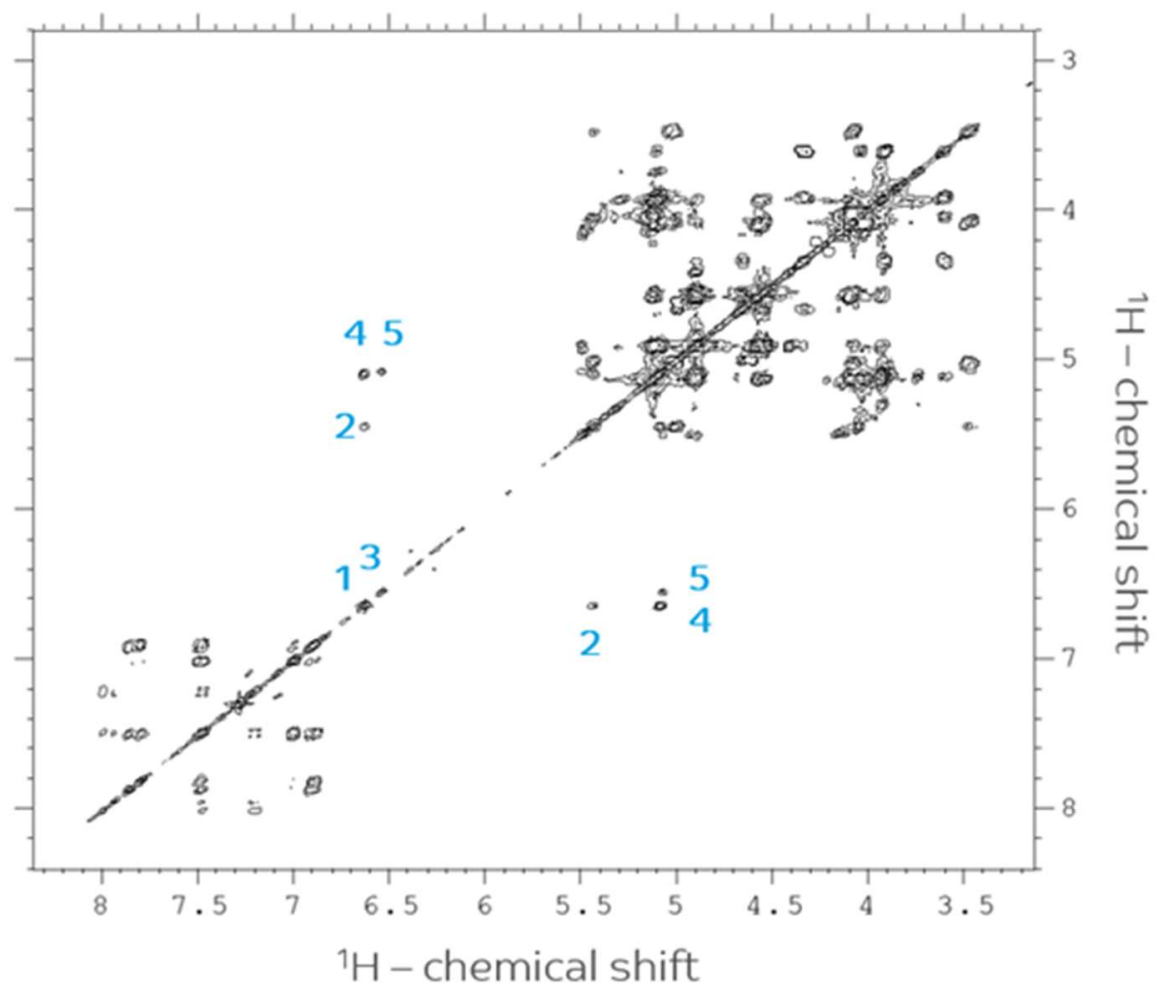

Figure SI-1. COSY NMR spectrum of isosorbide homopolycarbonate after exposure to heat (300 ${ }^{\circ} \mathrm{C}$ ) during extrusion and molding (with peak assignments referring to Figure 2). 


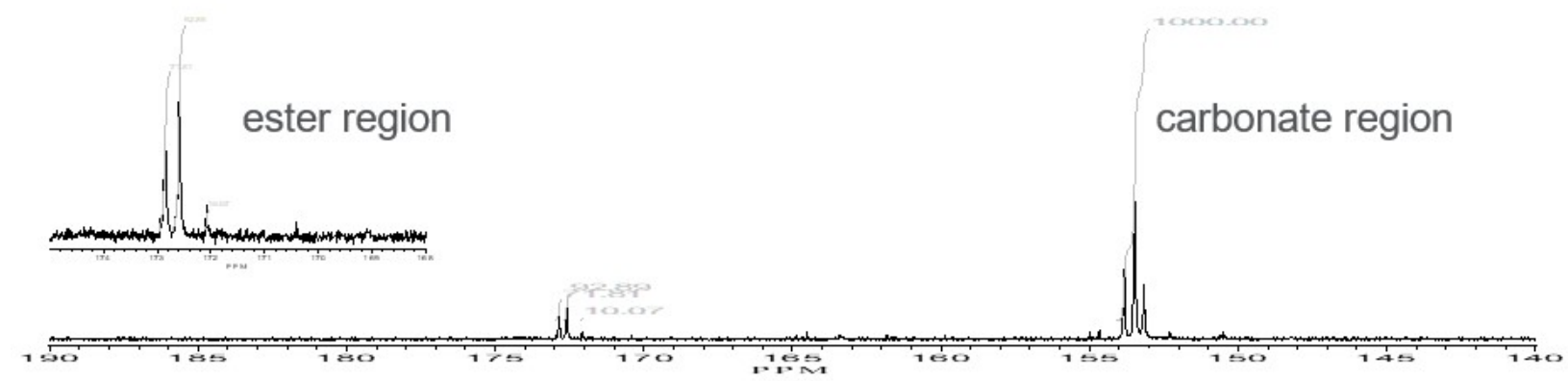

Figure SI-2. ${ }^{13} \mathrm{C}-\mathrm{NMR}$ spectrum of isosorbide - pri-1009 polycarbonate (S3), using the peak shifts in carbonate (isosorbide) and ester (pri-1009) region for calculating monomer content, coming to $7 \mathrm{~mole} \%$ for this example (20 $\mathrm{wt} \%)$
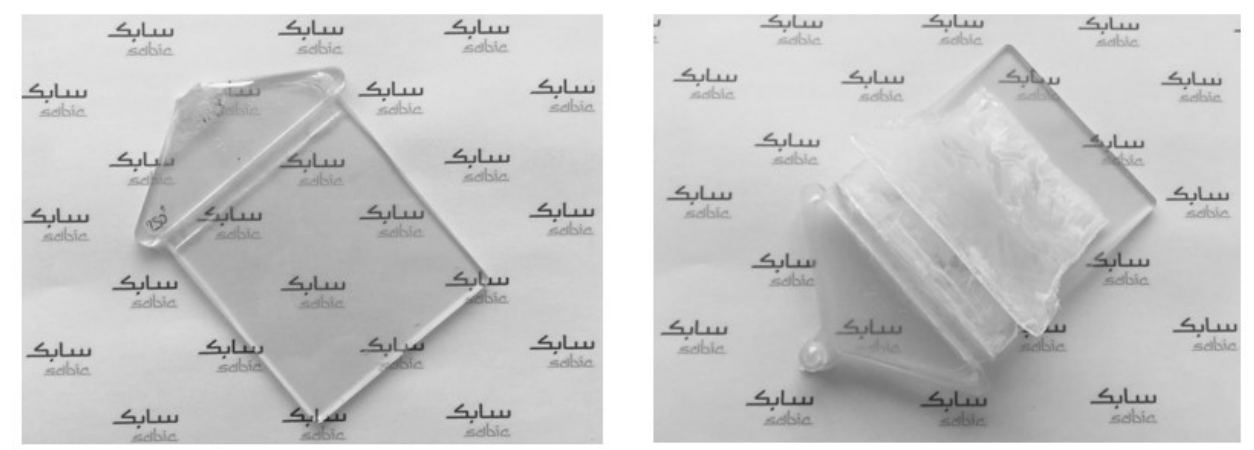

Figure SI-3. Figure 6. Images of color plaques produced from S5 (66.9\%, haze 15.3\%, measured at $2.5 \mathrm{~mm}$ ), showing delamination on the right where a layer was peeled off from the plaque.

Table SI-1. 13C-NMR integrals for determination of copolymer blockiness, normalized to 1000 on the carbonate integral (152-154 ppm)

\begin{tabular}{|c|c|c|c|c|}
\hline Sample & $\begin{array}{c}\text { Integral } \\
\text { carbonate }\end{array}$ & Integral IS-IS & Integral IS-pri & Integral pri-pri \\
\hline S3 & 1000 & 1000 & 164 & \\
\hline S5 & 1000 & 964 & 35 & 1 \\
\hline
\end{tabular}


<smiles>[R]C(=O)OC=[Po]C(C)C(C)C</smiles>

Scheme SI-2. Generalized polycondensation reaction between carbonate and acid, followed by decarboxylation resulting in ester

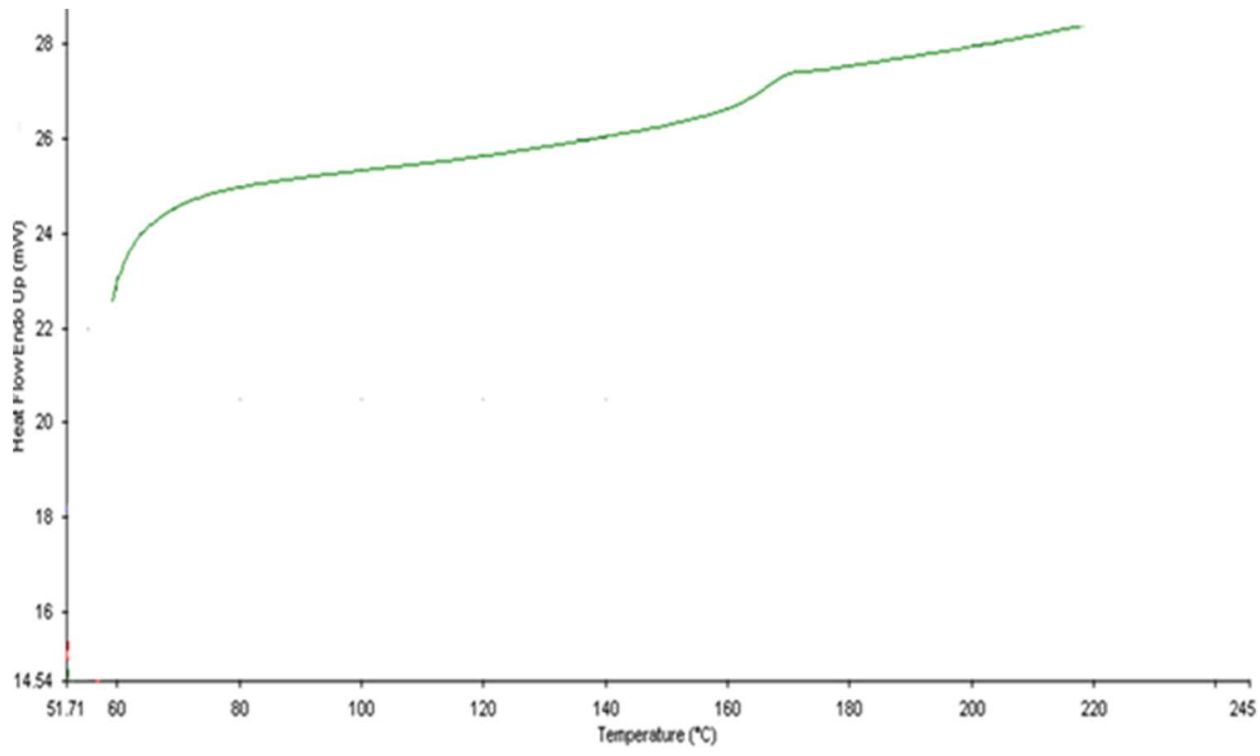

Figure SI-4. DSC plot of isosorbide - pri-1838 copolycarbonate (S5)

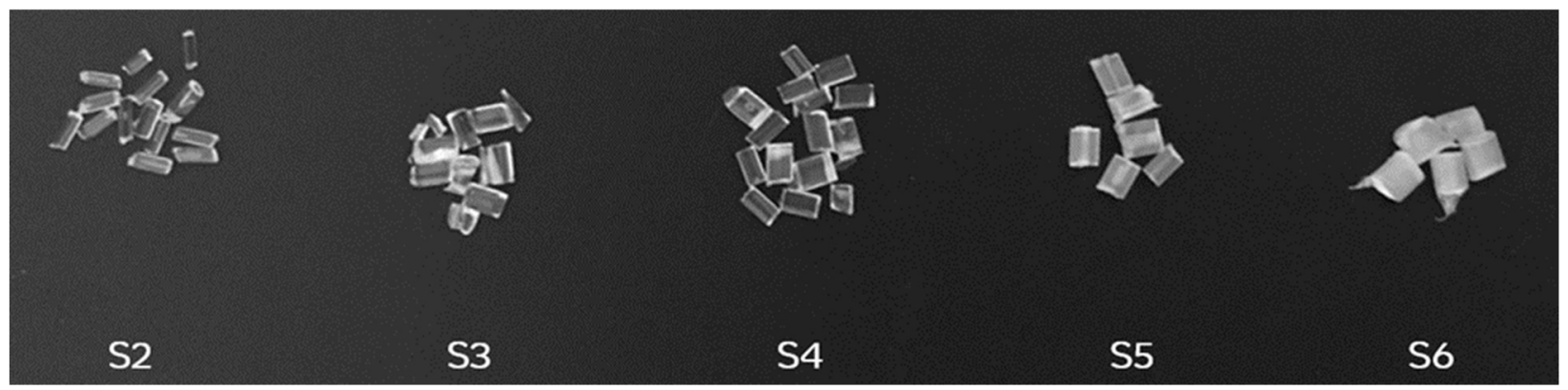

Figure SI-5. Pellets of isosorbide copolymers displaying haziness (S5, S6), where the isosorbide homopolymer and the samples with lower $T_{\mathrm{g}}$ are clear $(\mathrm{S} 2, \mathrm{~S} 3, \mathrm{~S} 4)$ 


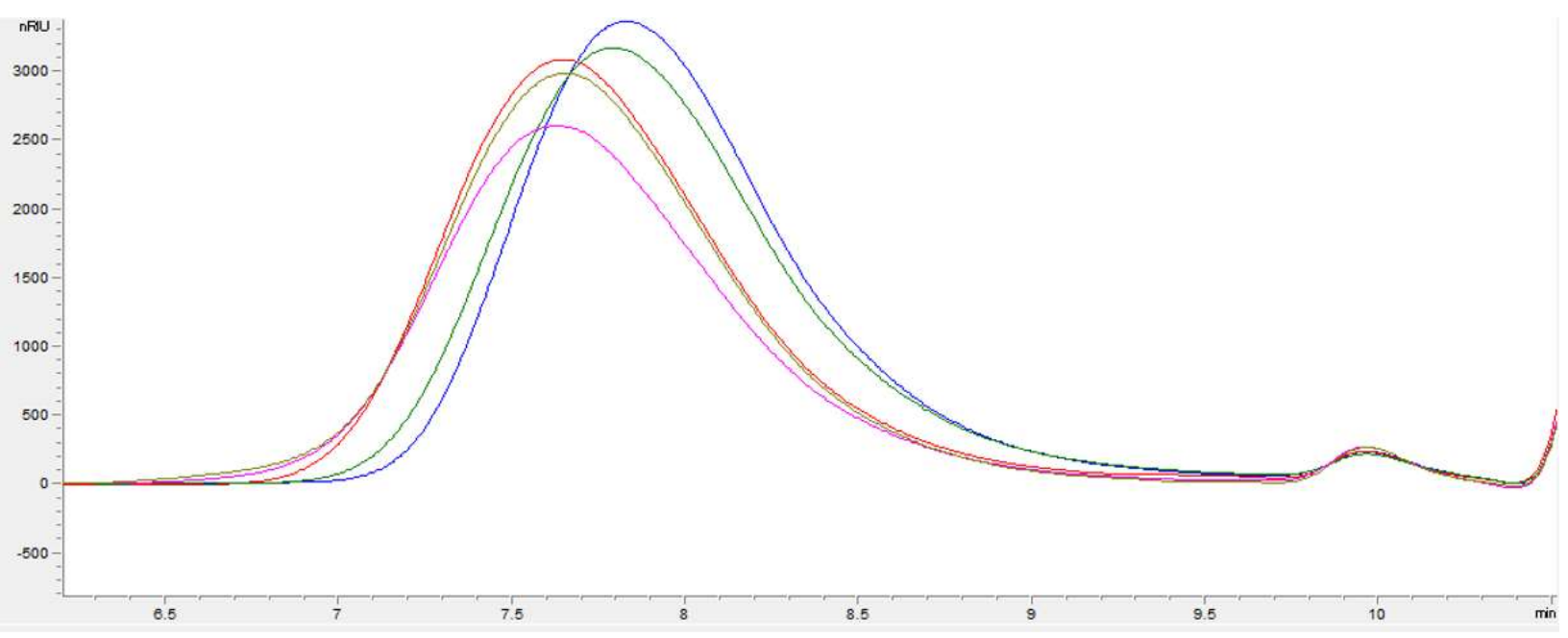

Figure SI-6. SEC chromatogram of isosorbide copolymers (using refractive index detector), $M_{\mathrm{w}}$ is calculated from these chromatograms using off line calibration line based on polystyrene standards 
a) $\mathrm{S} 17$

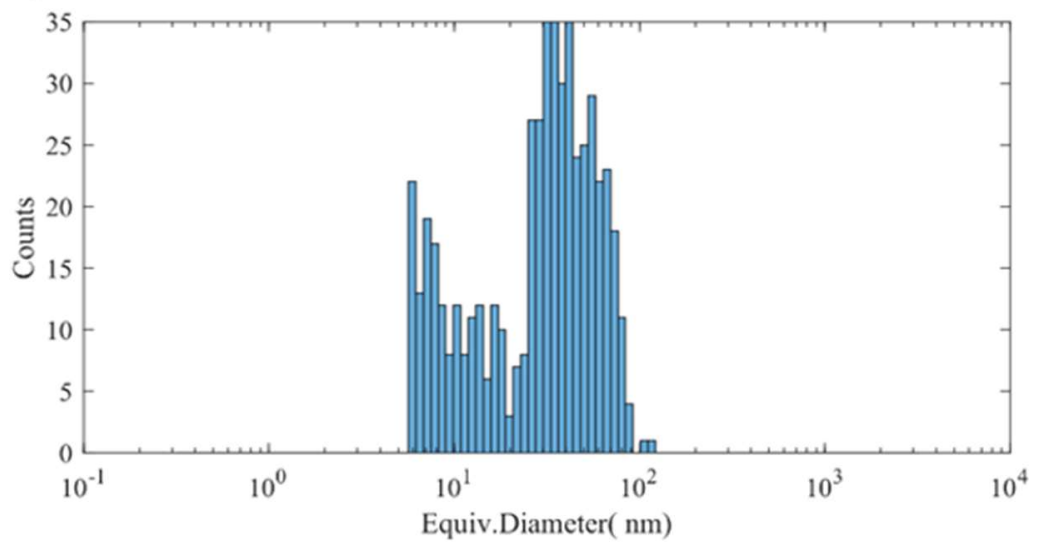

b) S18

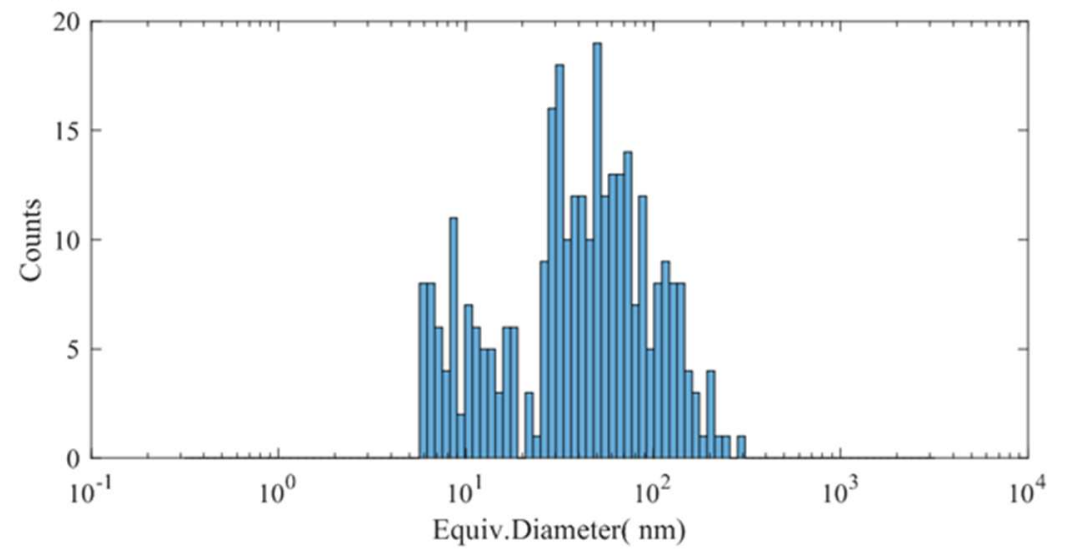

c) $\mathrm{S} 19$

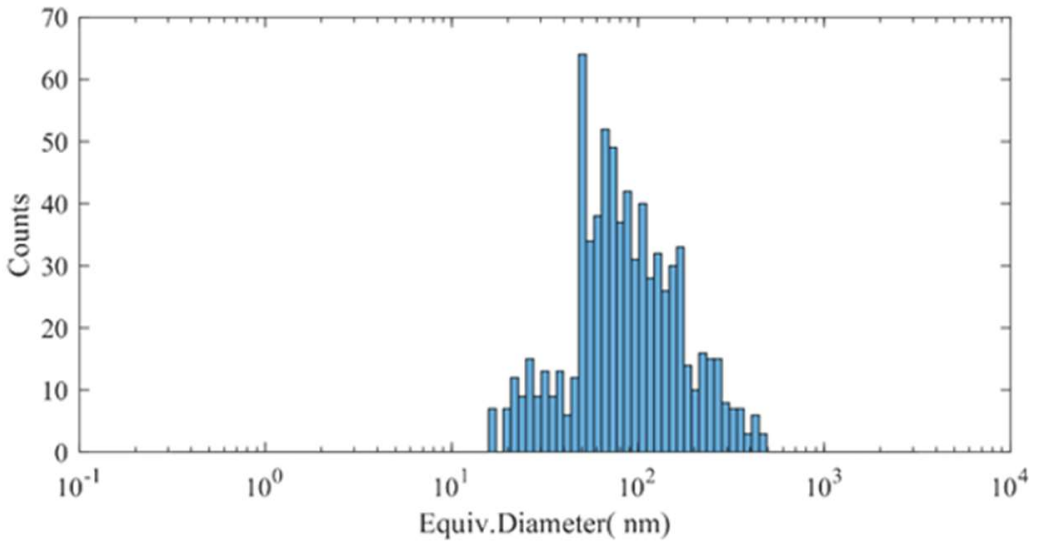

Figure SI-7. Data analysis of TEM micrographs for domain size distributions (equivalent diameter, nm), showing bimodal distributions for S17 (a) and S18 (b). 
Table SI-2. Statistical distribution of domain sizes (equivalent diameter, $\mathbf{n m}$ ) based on TEM micrographs

\begin{tabular}{c|ccc} 
& $\mathrm{S} 17$ & $\mathrm{~S} 18$ & $\mathrm{~S} 19$ \\
\hline Mean (Ln) & 34.2622 & 55.5007 & 107.9666 \\
Std.dev & 21.3805 & 47.3343 & 80.4352 \\
$\mathrm{~N}$ & 527 & 311 & 752 \\
& & & \\
$100 \%(\max )$ & 113.20 & 290.61 & 477.18 \\
$99.5 \%$ & 90.54 & 253.43 & 441.18 \\
$97.5 \%$ & 78.90 & 184.11 & 339.14 \\
$90 \%$ & 65.04 & 121.23 & 217.09 \\
$75 \%$ & 48.10 & 74.63 & 136.53 \\
$50 \%$ & 32.40 & 43.17 & 82.61 \\
$25 \%$ & 13.81 & 20.88 & 55.25 \\
$10 \%$ & 7.46 & 8.71 & 33.69 \\
$2.5 \%$ & 5.90 & 6.05 & 22.34 \\
$0.5 \%$ & 5.90 & 5.90 & 16.09 \\
$0.0 \%$ & 5.90 & 5.90 & 16.09
\end{tabular}




\section{DOE analysis}

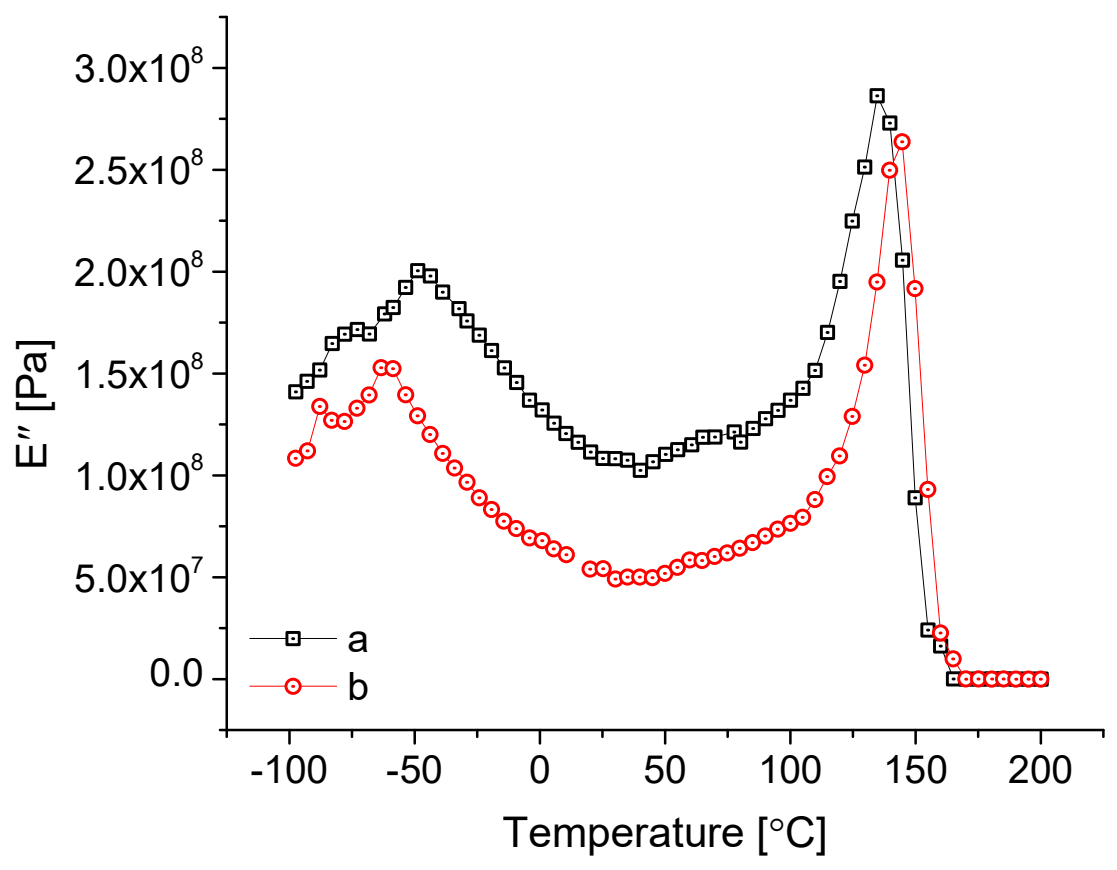

Figure SI-8. DMA comparison (E") of (a) S16 (low pri-1838), showing a $180{ }^{\circ} \mathrm{C}$ gap between the first $T_{\mathrm{g}}\left(-45^{\circ} \mathrm{C}\right)$ and the second $T_{\mathrm{g}}\left(135^{\circ} \mathrm{C}\right)$ and (b) S12 (high pri-1838), showing a $210^{\circ} \mathrm{C}$ gap between the first $T_{\mathrm{g}}\left(-65^{\circ} \mathrm{C}\right)$ and the second $T_{\mathrm{g}}\left(145^{\circ} \mathrm{C}\right)$, indicating more phase separation with increasing amount of pri-1838.

Table SI-3. Analysis of variance for the DOE factors (uncoded), only the significant factors $(95 \%$ confidence, $\mathbf{p}<\mathbf{0 . 0 5})$ are listed. For $\mathrm{C} M_{\mathrm{w}}$ after molding is used.

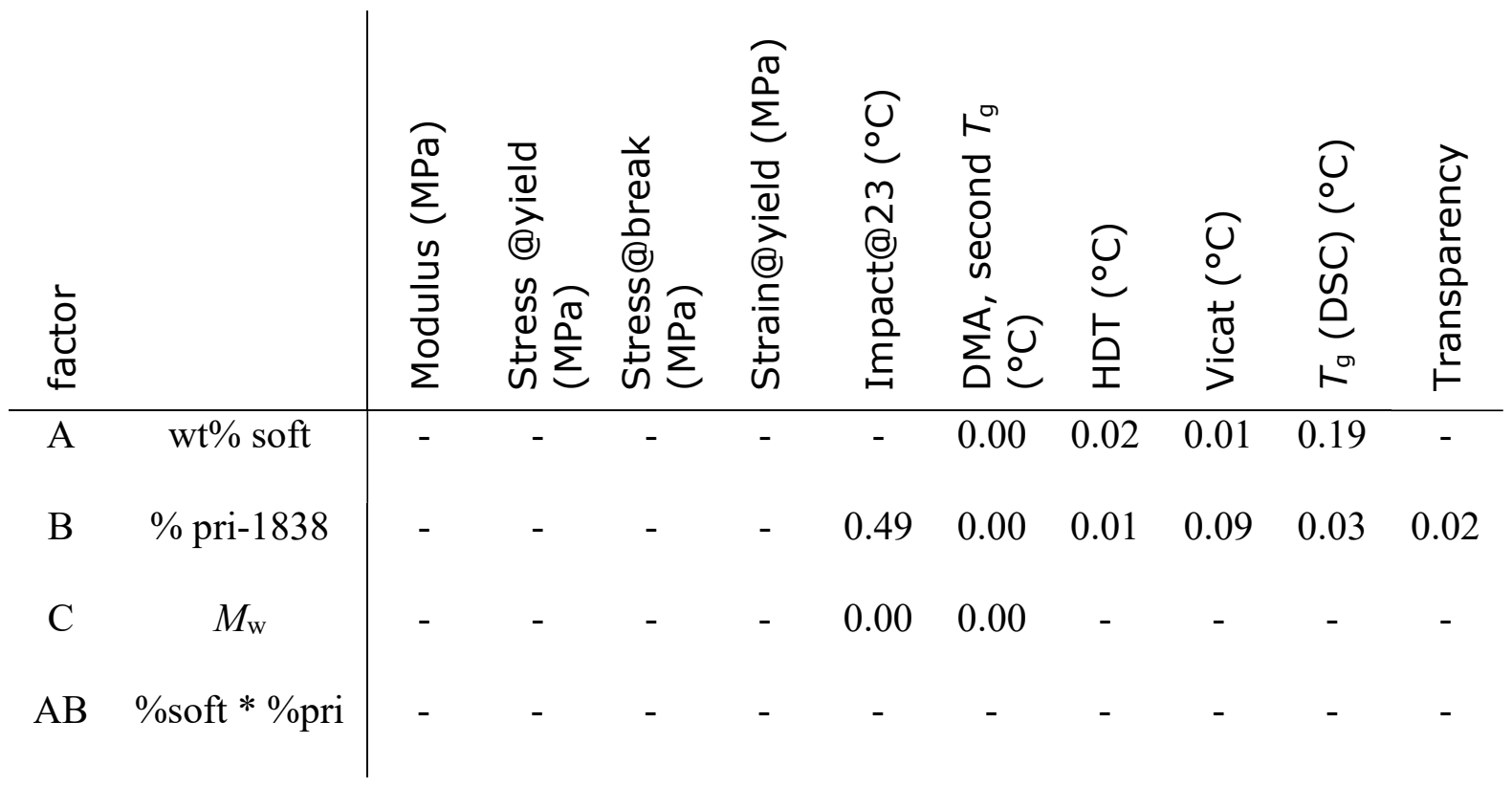




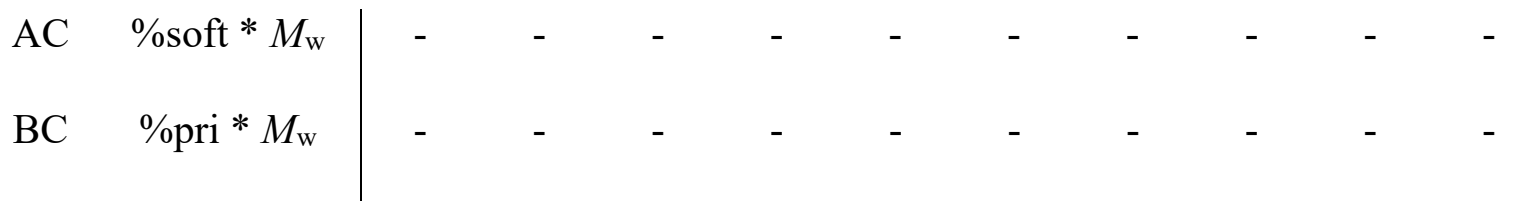

Table SI-4. Regression equations based on the DOE analysis (uncoded), including model significance (95\% confidence). Two way interactions were only included if the extended model was significant, else only single interactions reported.

\begin{tabular}{l|l|c} 
property & Regression Equation & p-value \\
\hline Modulus & $=3781-23.1 \%$ soft $+1.59 \%$ pri-1838-14.66 $M_{\mathrm{w}}$ & 0.109 \\
Stress@yield & $=108.1-0.375 \%$ soft $+0.1372 \%$ pri-1838-0.565 $M_{\mathrm{w}}$ & 0.100 \\
Stress@break & $=91.2-0.915 \%$ soft $+0.011 \%$ pri-1838-0.419 $M_{\mathrm{w}}$ & 0.182 \\
Strain@yield & $=3.23+0.221 \%$ soft $-0.0324 \%$ pri-1838 $0.0766 M_{\mathrm{w}}$ & 0.201 \\
Impact & $=-39.4-0.159 \%$ soft $+0.1812 \%$ pri-1838 $+1.546 M_{\mathrm{w}}$ & 0.007 \\
DMA 2 ${ }^{\text {nd }} T_{\mathrm{g}}$ & $=150.04-0.622 \%$ soft $+0.1935 \%$ pri-1838+0.163 Mw & 0.000 \\
HDT & $=108.56-1.062 \%$ soft $+0.274 \%$ pri-1838 $-0.030 M_{\mathrm{w}}$ & 0.001 \\
Vicat & $=144.74-1.284 \%$ soft $-0.1453 \%$ pri-1838 $+0.415 M_{\mathrm{w}}$ & 0.036 \\
$T_{\mathrm{g}}$ & $=139.92-0.631 \%$ soft $+0.1902 \%$ pri-1838 $+0.240 M_{\mathrm{w}}$ & 0.008 \\
$\% \mathrm{~T}$ & $=96.9+2.01 \%$ soft $-0.537 \%$ pri-1838 $-0.537 M_{\mathrm{w}}$ & 0.039
\end{tabular}
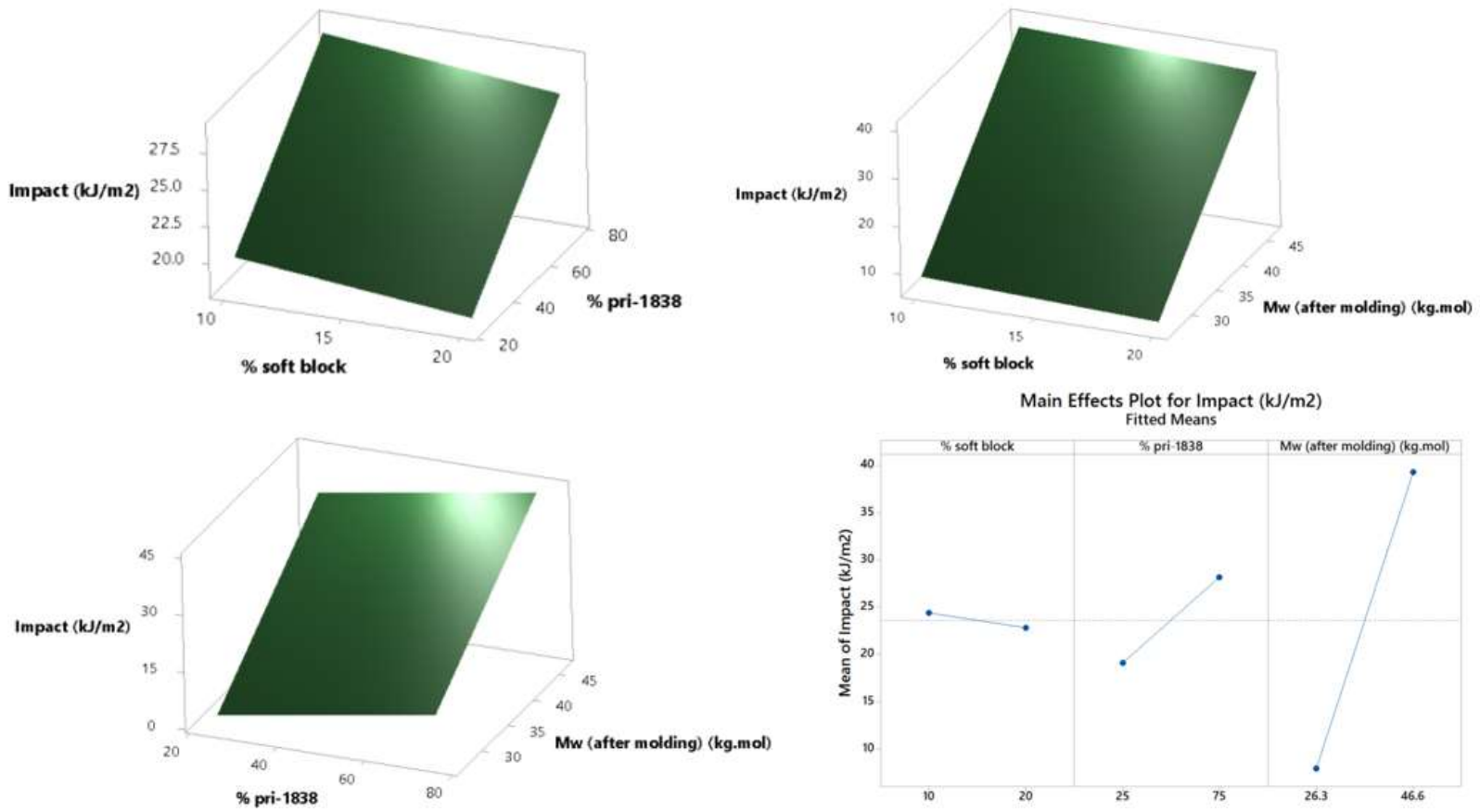

Figure SI-9. Main effect and surface plot of the terms on impact (in $\mathrm{KJ} / \mathrm{m}^{2}$ ) 

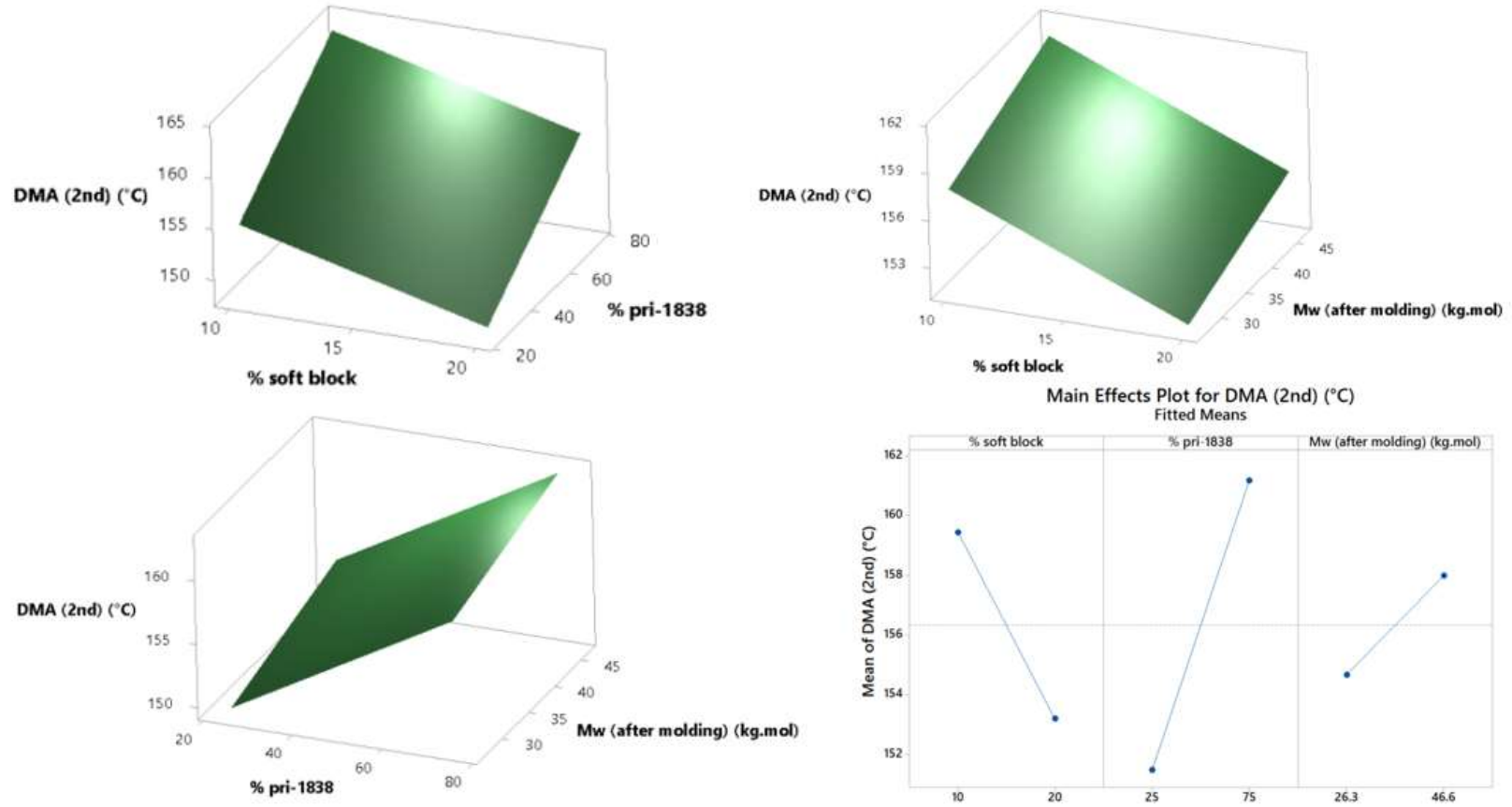

Figure SI-10. Main effect and surface plot of the terms on high temp $T_{\mathrm{g}}$ measured in DMA
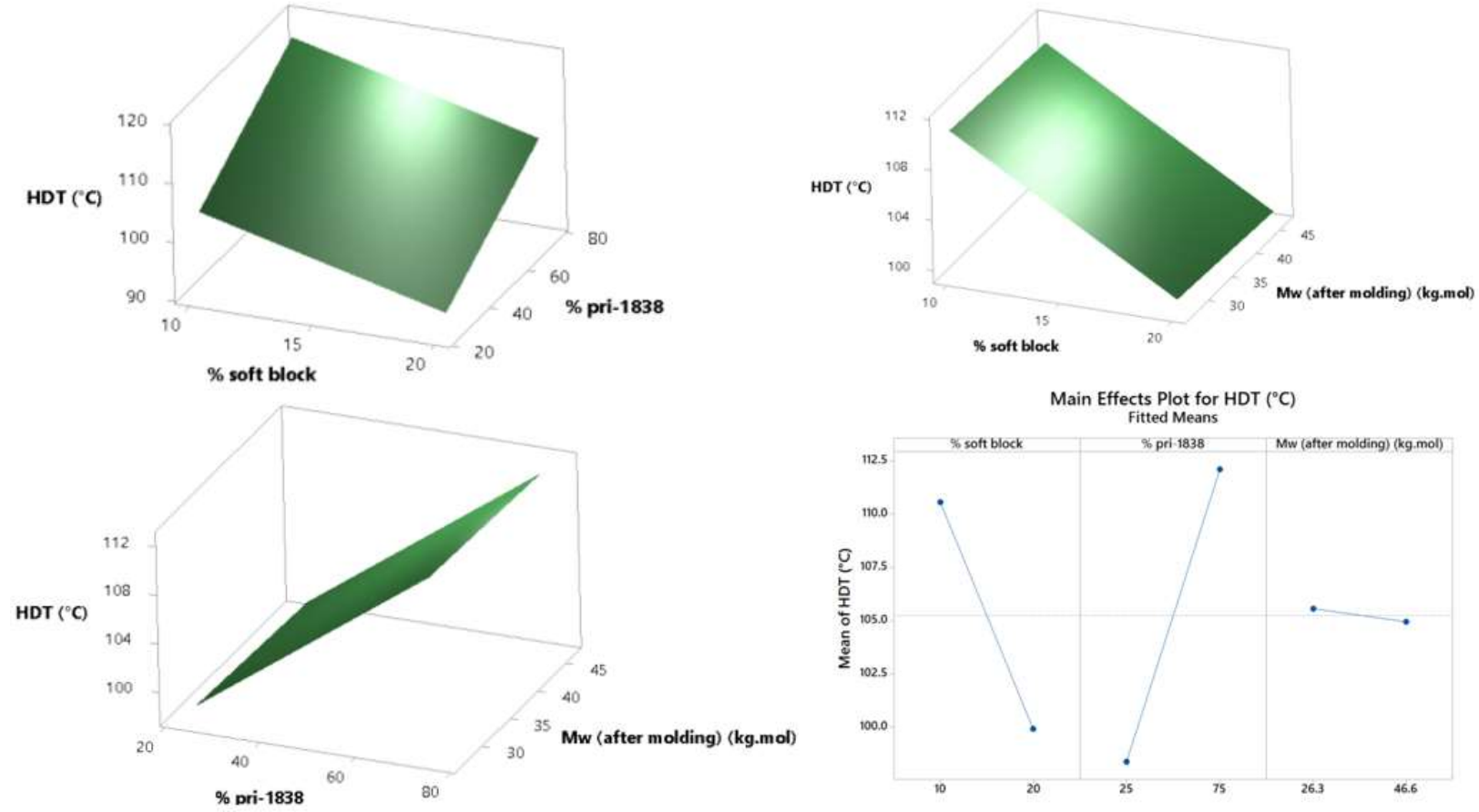

Figure SI-11. Main effect and surface plot of the terms on HDT $\left({ }^{\circ} \mathrm{C}\right)$ 

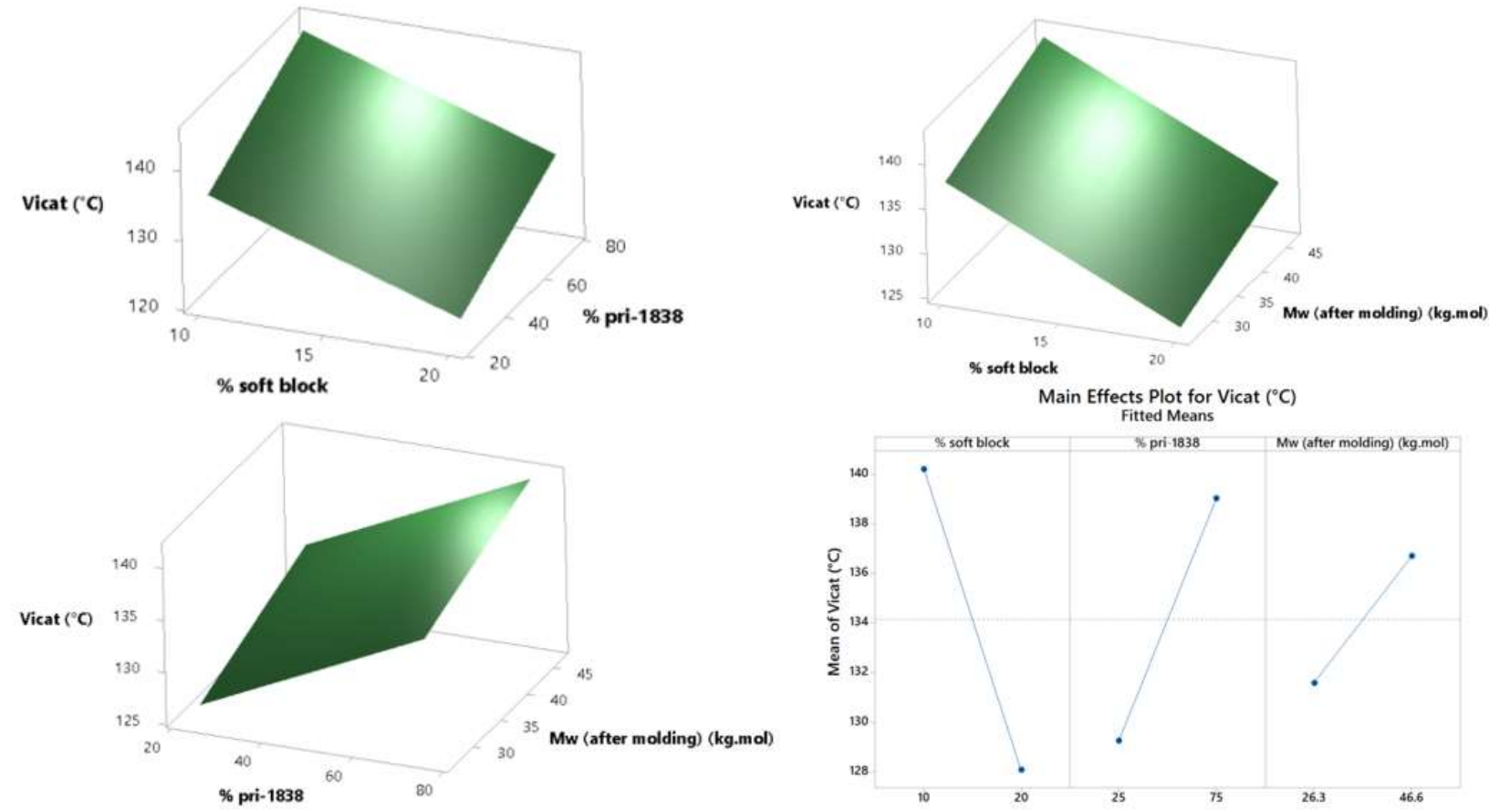
Main Effects Plot for Vicat $\left({ }^{\circ} \mathrm{C}\right)$

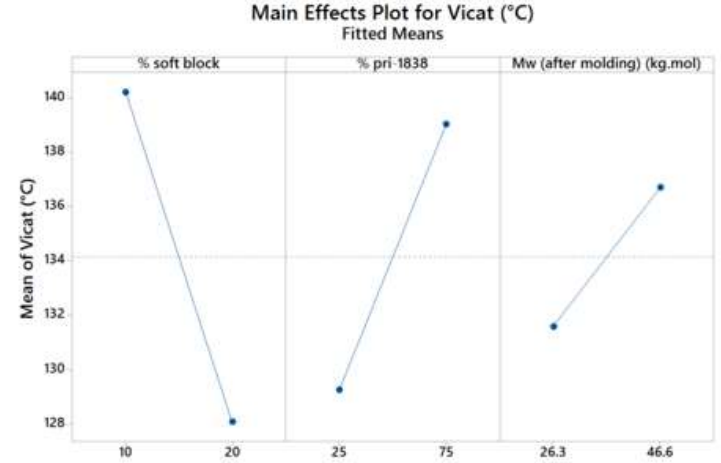

Figure SI-12. Main effect and surface plot of the terms on vicat $\left({ }^{\circ} \mathrm{C}\right)$
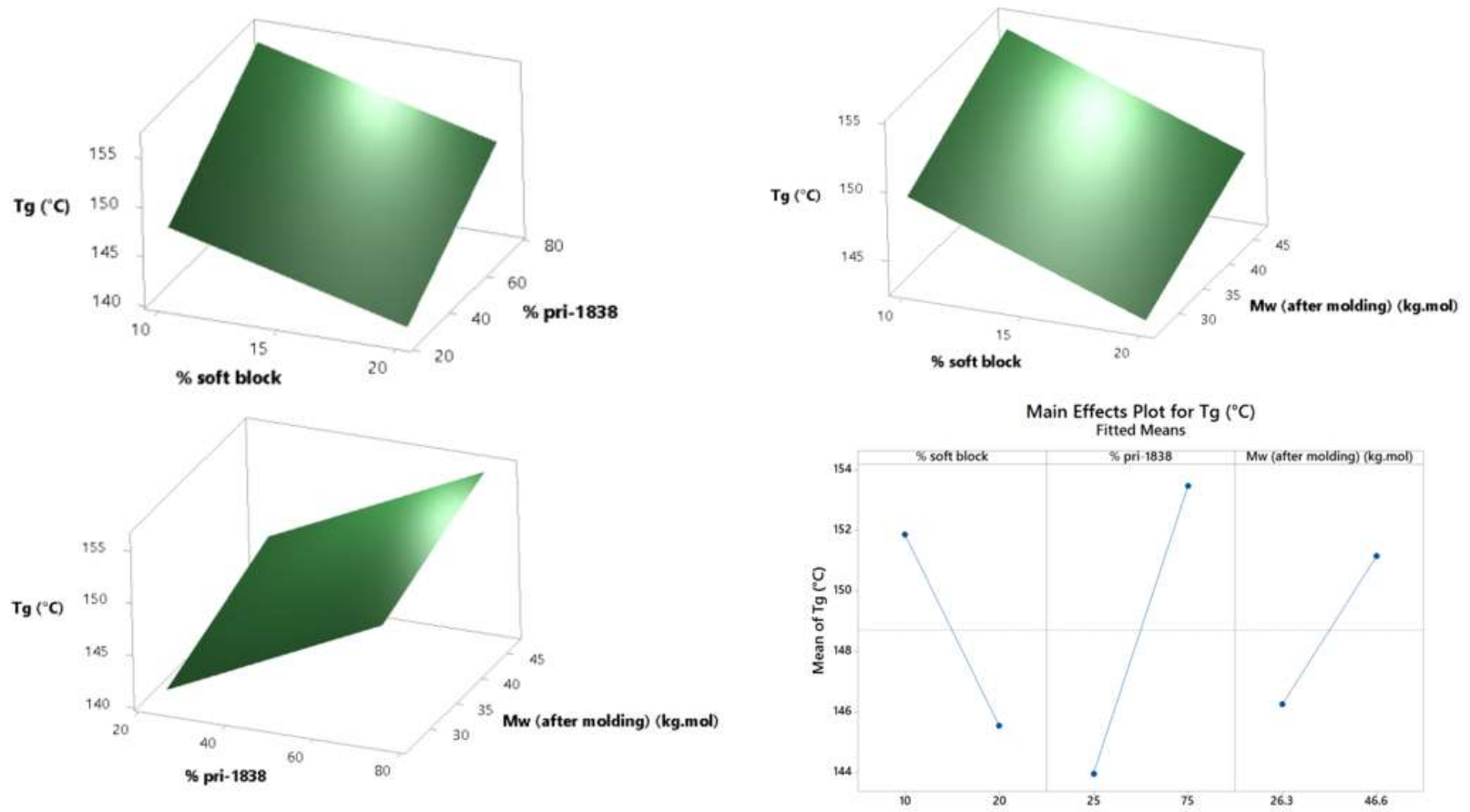

Figure SI-13. Main effect and surface plot of the terms on $T_{\mathrm{g}}\left(\right.$ in $\left.{ }^{\circ} \mathrm{C}\right)$ 

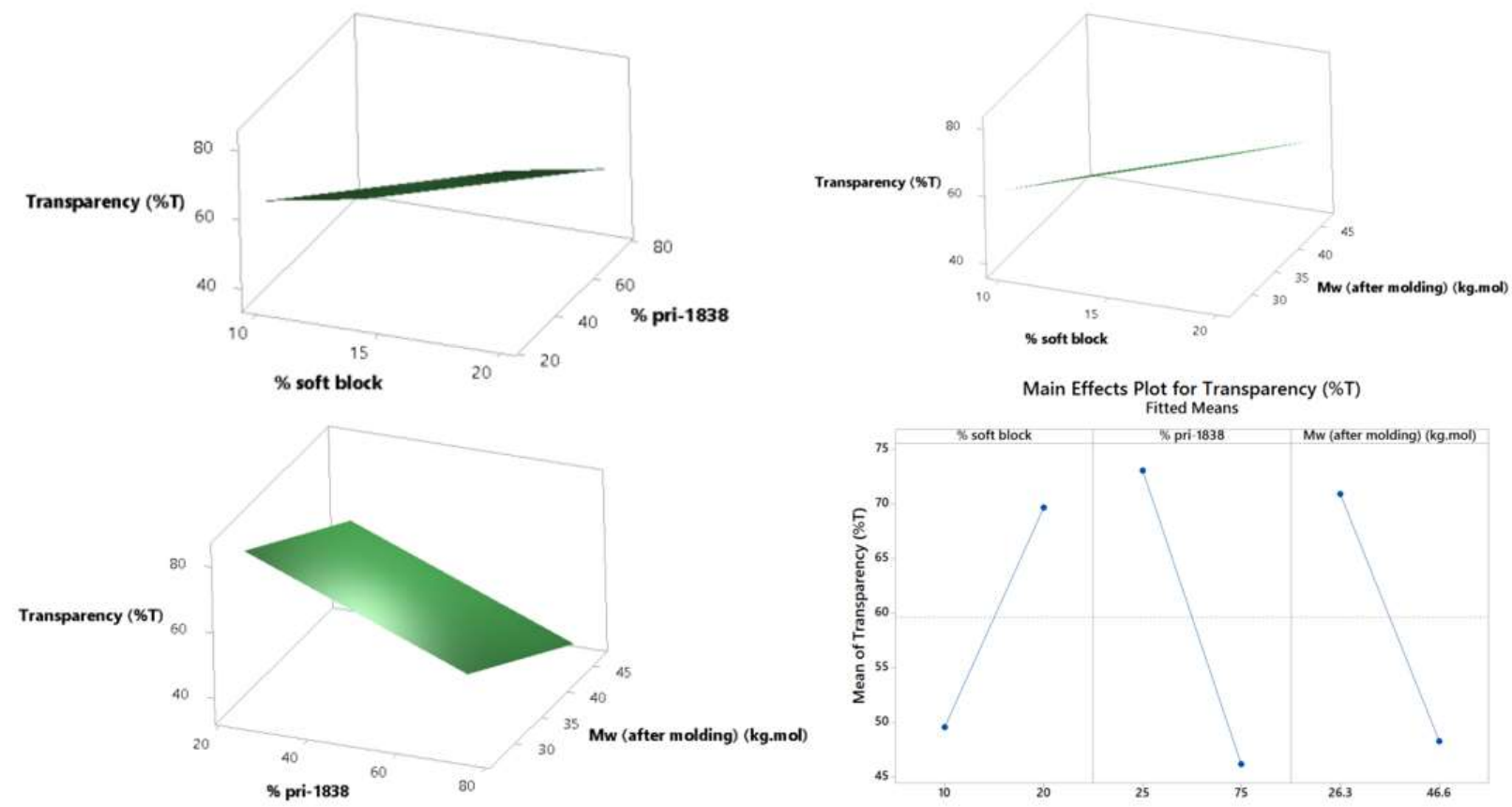

Figure SI-14. Main effect and surface plot of the terms on transparency (in \%)

\section{Rheology}

Introduction of a co-monomer affects the stiffness of the chain. This could have consequences in rheological and mechanical properties. For example, addition of a stiff monomer can result in the entanglement molecular weight increasing. A measure for the chain stiffness is the characteristic ratio $\mathrm{C}_{\infty}$. This is calculated based on the model proposed by $\mathrm{Wu}^{1,2}$ from melt rheology experiments as shown by eq. 1 .

$$
C_{\infty}=\left(\frac{\rho R T}{2 M_{v} G_{N}^{0}}\right)^{1 / 2}=\frac{\left\langle r_{0}{ }^{2}\right\rangle}{n l^{2}}
$$

In the above equation, $G_{N}^{0}$ is the rubbery plateau modulus, where $G_{N}^{0}=G^{\prime}(\omega)_{\tan \delta \rightarrow \min }, G^{\prime}(\omega)$ values are obtained from the master curve, $\rho$ represents the density $\left(\mathrm{g} / \mathrm{cm}^{3}\right), R$ the universal gas constant, $T$ the absolute temperature $(\mathrm{K}), r_{0}{ }^{2}$ is the ratio of the mean end-to-end distance of the real chain to that of an unperturbed chain with equal contour length, $\mathrm{n}$ is the number of statistical segments, $l$ is length of each statistical element, and $M_{v}$ being the molecular weight of a skeletal unit. 
1. $\mathrm{S}$. $\mathrm{Wu}$, Secondary relaxation, brittle-ductile transition temperature, and chain structure, J. Appl. Polym. Sci. 1992, 46, 619-624.

2. R. Wimberger-Friedl, H. F. M. Schoo, Chain Stiffness of Copolycarbonates Containing a Spiro Linkage, Macromolecules 1996, 29, 8871-8874. 